\title{
Freezing as a method of sample preservation for the analysis of dissolved inorganic nutrients in seawater
}

\author{
John E. Dore, Terrence Houlihan, Dale V. Hebel, Georgia Tien, Luis Tupas, \\ David M. Karl \\ Department of Oceanography, School of Ocean and Earth Science and Technologv, Unilersity of Hawaii, Honolulu, HI 96822, USA
}

Received 6 February 1995; accepted 15 December 1995

\begin{abstract}
It is often desirable or necessary to store collected seawater samples prior to analysis for dissolved inorganic nutrients. It is therefore important to establish preservation and storage techniques that will ensure sample integrity and will not alter the precision or accuracy of analysis. We have performed a series of experiments on the storage of nutrient samples collected at the oligotrophic North Pacific benchmark Station ALOHA, using both standard autoanalyses and low-level techniques. Our results reveal that for oligotrophic occanic waters, the immediate freezing of an unfiltered water sample in a clean polyethylene bottle is a suitable preservation method. This procedure is simple, it avoids potentially contaminating sample manipulations and chemical additions, and it adequately preserves the concentrations of nitrate + nitrite, soluble reactive phosphate, and soluble reactive silicate within a single water sample.
\end{abstract}

\section{Introduction and background}

Accurate measurements of dissolved nutrient concentrations in seawater are essential to the goals of many oceanographic research programs. Because the concentrations of the major nutrients in unpreserved seawater samples are altered, sometimes rapidly, by physical, chemical and biological processes, such samples must be analyzed immediately or preserved in a manner that successfully maintains the original concentrations of the species of interest until analyses can be performed. Unfortunately, the nature of oceanographic research often precludes immediate at-sea chemical determinations from being made, whether due to heavy seas, inadequate laboratory space, improper electric power conditioning, hazard of contamination, lack of capable personnel or any of a myriad of other difficulties potentially encountered aboard a research vessel. Even when these problems can be solved, it may still be undesirable to perform at-sea analyses if the potential errors introduced as a result of working in a shipboard environment exceed the potential errors introduced through sample preservation and storage. It is therefore of great interest to marine chemists to find suitable preservation and storage methods for each of the major nutrients in seawater.

A successful preservation technique must maintain the original concentration of an analyte during storage to within the level of accuracy required by the particular program. Beyond this absolute requirement, there are a number of additional criteria to evaluate, including simplicity, relative ease of operation and rapid implementation. The ideal method 
would not require exposure of the sample to potentially contaminating objects or environments. If possible, chemical additions to the sample would be avoided. Finally, the optimal method will preserve all of the constituents of interest in a single sample without requiring different procedures for different chemical species.

For decades marine chemists have tested the suitability of various preservation techniques, considering the potcntial effects of filtration, storage container, temperature, chemical additions and radiation on samples of different water types. The considerable body of literature produced presents varied and often contradictory opinions on the effectiveness of preservation methods. Freezing of water samples in polyethylene containers is a very popular method, as it holds all of the desirable qualities listed above. Nevertheless, there is not total agreement as to whether freezing maintains the original nutrient concentrations within a sample to an acceptable degree of accuracy.

Recently we reviewed the existing literature on the frozen storage of nutrient samples in natural waters, finding 41 such references between 1953 and 1995 (Dore, 1995). Many offer recommendations without data, deal with fresh or estuarine waters, or test nutrients other than the three considered here. Only 11 of the 41 references located present actual data on the frozen storage of seawater nitrate + nitrite $(\mathrm{N}+\mathrm{N})$, soluble reactive phosphate (SRP) and/or soluble reactive silicate (SRSi) samples (Collier and Marvin, 1953; Charpiot, 1969; Burton et al., 1970; Ryle et al., 1981; Morse et al., 1982; Venrick and Hayward, 1985; Kremling and Wenck, 1986; Macdonald et al., 1986; Chapman and Mostert, 1990; Sakamoto et al., 1990; Clementson and Wayte, 1992). Two of these studies (Morse et al., 1982; Venrick and Hayward, 1985) conclude that none of the three nutrients considered here is adequately preserved by freezing; we will discuss these two investigations separately from the others.

We suspect that the experiments conducted by Morse et al. (1982) are not representative of the true effects of freezing on seawater nutrient samples, possibly due to "unknown" quality control problems. It is difficult to explain how the $[\mathrm{N}+\mathrm{N}]$ of replicate samples stored in polyethylene bottles at $-20^{\circ} \mathrm{C}$ can increase from 20 to $44 \mu M$ after one day, then decrease to $32 \mu M$ after six more days (Morse et al., 1982). These extreme changes are an order of magnitude greater than any changes observed under similar conditions by other investigators. The study by Venrick and Hayward (1985), on the other hand, does not appear to be affected by such extreme variability in concentrations. Although the latter authors do not recommend frozen storage of seawater nutrient samples, they do state that "When the data from all nutrients are weighted equally and combined, the most accurate and precise storage method is freezing." Their recommendation against frozen storage appears to be due mainly to relatively large errors at the lowest concentrations tested. For this reason, we will pay particular attention to the low end of the concentration scales, especially in regard to $\mathrm{N}+\mathrm{N}$ and SRP, for which we have low-level analytical methods at our disposal.

Of the remaining 9 studies above, 5 test the effects of freezing on $[\mathrm{N}+\mathrm{N}]$, and all of these conclude that this nutrient is adequately preserved by freezing (Dore, 1995). Similarly, 5 of the 6 studies dealing with SRSi recommend freezing for sample preservation, except for samples with [SRSi] above some threshold, which is reported as being anywhere between 30 and $70 \mu \mathrm{M}$, depending on the particular investigation (Macdonald et al., 1986; Chapman and Mostert, 1990). This "silicate problem" is apparently due to the formation of polymerized forms at high concentrations during freezing, but it has been pointed out that prolonged thawing results in the recovery of these polymerized forms as SRSi (Walsh, 1989; Sakamoto et al., 1990). The preservation of [SRP] by freezing is more of a matter of debate, with 4 of 6 studies recommending freezing but 2 citing increases in [SRP] variability after freezing (Charpiot, 1969; Chapman and Mostert, 1990).

In this paper we will focus on the freezing method for the preservation of the concentrations of $\mathrm{N}+\mathrm{N}$, SRP and SRSi in water column samples collected at the oligotrophic North Pacific Station ALOHA; we have reported the results of similar experiments on nitrite $\left(\mathrm{NO}_{2}^{-}\right)$elsewhere (Dore and Karl, 1996). Oligotrophic regions of the ocean are characterized by low standing stocks of biomass, low particulate loads and low surface nutrient concentrations. At Station ALOHA, chlorophyll- $a$ concentrations seldom exceed $0.3 \mathrm{mg} \mathrm{m}^{-3}$, total particulate loads are 
usually $<50 \mathrm{mg} \mathrm{m}^{-3}$ and surface nutrient concentrations are usually $<15 \mathrm{n} M$, $<100 \mathrm{n} M$ and $<2$ $\mu M$ for $\mathrm{N}+\mathrm{N}, \mathrm{SRP}$ and SRSi, respectively. Oligotrophic regions encompass $>75 \%$ of the total area of the global ocean (Lewis et al., 1986) and are responsible for $>80 \%$ of oceanic primary production (Martin et al, 1987; Karl et al., 1996). Because of the areal extent of these waters, accurate and precise measurements of their nutrient concentrations are essential to understanding the biogeochemical cycles of the major bioelements.

\section{Materials and methods}

\subsection{Sample collection}

The Hawaii Ocean Time-series (HOT) project has maintained a deep water oceanic station dubbed ALOHA (A Long-term Oligotrophic Habitat Assessment; Karl and Lukas, 1996) since October 1988. Station ALOHA, located at $22^{\circ} 45^{\prime} \mathrm{N}, 158^{\circ} \mathrm{W}$, is occupied at approximately monthly intervals for the collection of physical, chemical and biological data relevant to the goals of the U.S.-JGOFS and WOCE programs. Discrete water samples are collected from hydrocasts using a CTD-rosette system outfitted with 24 12-1 polyvinylchloride (PVC) sampling bottles with Teflon-coated springs. During each HOT cruise, samples for a suite of inorganic and organic constituents are collected over the entire $4750-\mathrm{m}$ water column.

All water samples analyzed for this study were collected at Station ALOHA during regular HOT cruises. Depending upon the individual experiment, samples analyzed for this study were collected either in 125- or 500-ml high-density polyethylene (HDPE) bottles, or in 4-1 polycarbonate (PC) bottles from which subsamples were decanted into individual HDPE bottles. Collection bottles were always acidwashed $(3 \times$ with $1 M \mathrm{HCl})$, rinsed $3 \times$ with deionized distilled water (DDW) and completely dried before use. Each bottle was also rinsed $3 \times$ with sample before collection. No samples were prefiltered except those indicated in the filtration experiments, which were vacuum filtered through acid-washed Whatman GF/ $F$ filters using polycarbonate filtration manifolds. All freezing was done in 125- or 500-ml HDPE bottles, filled $\sim \frac{2}{3}$ full and stored upright so as to prevent the seawater from freezing out around the screw-cap. Freezing was achieved by immediate placement of samples into a $-20^{\circ} \mathrm{C}$ walk-in or upright chest freezer. Samples remained frozen at $-20^{\circ} \mathrm{C}$ in the dark until $4-12 \mathrm{hr}$ before analysis, at which time they were completely thawed at room temperature in air or in a water bath, and allowed to achieve laboratory temperature.

\subsection{Analytical methods}

Most of the [N + N], [SRP] and [SRSi] analyses in this study were carried out on a 4-channel continuous flow Technicon Autoanalyzer II ${ }^{*}$ by Mr. Ted Walsh of Analytical Services, SOEST, University of Hawaii. The methods used follow those recommended by Technicon Industrial Systems (1973, $1977,1979)$ with only slight modifications to surfactant concentrations, sampling rate and pump tube size (T. Walsh, pers. commun.). These analytical techniques are adaptations of the $\mathrm{N}+\mathrm{N}$ method of Armstrong et al. (1967), the SRP method of Murphy and Riley (1962) and the SRSi method of Strickland and Parsons (1972). DDW is used as the diluent for all reagents and to establish the baseline. Seawater refractive index and turbidity blanks are used to correct each type of analysis. The SRP color reagent and all calibration standards are made fresh daily, the latter through dilution of high-concentration stocks with filtered low-nutrient seawater (surface water from Station ALOHA). Each standard is corrected for the absorbance of the seawater diluent and each sample is corrected for its blank absorbance at the appropriate wavelength. Accuracy was maintained through the use of internal standards and certified reference standards (CSK; Ambe, 1978). The detection limits and approximate analytical precisions for these autoanalyses are as follows: $N+N$, detection limit $=0.03 \mu M$, precision $= \pm 0.3 \%$; SRP, detection limit $=0.02 \mu M$, precision $= \pm 0.5 \% ;$ SRSi, detection limit $=0.3 \mu M$, precision $= \pm 1.5 \%$. The stated precisions are for samples with $\mathrm{N}+\mathrm{N}$, SRP and SRSi concentrations $>0.2,>0.4$ and $>2 \mu M$. respectively; for samples below these levels we estimate the precision to be on the order of the value of the detection limit.

Because we were not able to take the Analytical Services Technicon autoanalyzer and its operator out to sea, our comparison of samples analyzed fresh 
at-sea and after frozen storage was performed using a different instrument. For these experiments we used an Alpkem RFA $/ 2{ }^{\circledR}$ four-channel autoanalyzer and employed the methods recommended by the manufacturer (Alpkem Corporation, 1989a,b,c), except for slight modifications in selected pump tube flow rates. The $\mathrm{N}+\mathrm{N}$ method is an adaptation of that published in APHA (1976), the SRP method is adapted from that of Murphy and Riley (1962), and the SRSi method is adapted from that of Truesdale and Smith (1975). Standards were made up fresh daily from concentrated stock solutions, and accuracy was maintained using CSK standards and through intercomparisons with Analytical Services. Unfortunately, we were not able to achieve the same level of precision with the Alpkem instrument as that obtained with the Technicon instrument by Analytical Services. The average precisions of replicate analyses both at-sea and in the shore-based laboratory for samples with $\mathrm{N}+\mathrm{N}, \mathrm{SRP}$ and SRSi concentrations $>0.2,>0.4$ and $>2 \mu M$, respectively, were as follows: $\mathrm{N}+\mathrm{N}, \pm 1.1 \%$; SRP, $\pm 1.9 \%$; $\mathrm{SRSi}, \pm 2.4 \%$.

Selected samples for $\mathrm{N}+\mathrm{N}$ were also analyzed using the chemiluminescent technique developed by Cox (1980) and modified for seawater by Garside (1982). This method involves wet chemical reduction of the species of interest to nitric oxide with subsequent detection in a commercial chemiluminescent nitrogen detector (Antek model 720). No major modifications to the Garside procedure were used. The detection limit for $\mathrm{N}+\mathrm{N}$ has been estimated to be $\sim 1-2 \mathrm{n} M$, with a precision of $\pm 0.5 \mathrm{n} M$ for samples with $[\mathrm{N}+\mathrm{N}] \leq 50 \mathrm{n} M$ and $\pm 1.6 \%$ for higher concentrations (Dorc and Karl, 1996).

Selected samples were analyzed for [SRP] using the magnesium-induced coprecipitation (MAGIC) method developed by Karl and Tien (1992). This method involves the pre-concentration of SRP through coprecipitation with authigenic brucite $\left(\mathrm{Mg}(\mathrm{OH})_{2}\right)$, followed by analysis using the standard molybdenum blue color reaction. The detection limit is $\sim 1 \mathrm{n} M$ and the approximate precision of the analysis is $\pm 3 \%$.

\subsection{Experiments on the effects of freezing samples}

We performed a series of experiments to assess sample integrity of unfiltered seawater samples dur- ing a freeze-thaw cycle. Samples were collected from ten depths at Station ALOHA between 5 and $4500 \mathrm{~m}$ into replicate HDPE bottles. One set was immediately placed into the freezer and the other was analyzed fresh at-sea for [N + N], [SRP] and [SRSi] using the Alpkem autoanalyzer. The frozen samples were returned to the shore-based laboratory and analyzed one week later using the same instrument.

In the low-level $\mathrm{N}+\mathrm{N}$ experiment, samples were taken from a number of depths from $0-150 \mathrm{~m}$ into replicate HDPE bottles. One set was immediately placed into the freezer and the other was analyzed at-sea using the chemiluminescent method. All at-sea analyses were completed within $8 \mathrm{hr}$ of collection, and during this time the bottles were stored in the dark at $4^{\circ} \mathrm{C}$. Frozen samples were analyzed 26 days later at our shore-based laboratory.

The low-level SRP experiment was performed in a similar manner, except fresh analyses were not performed at-sea. Several depths at Station ALOHA were sampled and from each a 4-1 polycarbonate bottle was filled. These bottles were maintained in the dark at $4^{\circ} \mathrm{C}$ until the day of the fresh analyses, at which time triplicate subsamples were taken in HDPE bottles. Two sets were then frozen immediately and the third analyzed using the MAGIC procedure. The frozen sets were analyzed 1 week and 2 weeks later. Because no at-sea determinations were made, the phosphate concentrations in these samples cannot be related to the in situ values. However, because the fresh analyses occurred immediately upon sample splitting, the results accurately reflect the potential freezing effects on SRP determinations.

Statistical analysis of each of the experiments on freezing samples was accomplished using Model II reduced major axis (geometric mean) linear regression following the recommendations of Ricker (1973, 1975). The confidence intervals of the slope and $y$-intercept were calculated based on the recommendations of Jolicoeur and Mossimann (1968) and York (1966), respectively.

\subsection{Fxperiments on the filtration of samples}

We performed experiments on $\mathrm{N}+\mathrm{N}$, SRP and SRSi to determine if filtration resulted in any differences in the frozen sample results. Samples were 
vacuum filtered through acid-washed $47-\mathrm{mm}$ glass fiber filters (Whatman $\mathrm{GF} / \mathrm{F}$ ) into acid-washed DDW-rinsed polycarbonate filtration manifolds. Filtered sample was used to rinse the manifold reservoir $3 \times$, then to rinse the HDPE sample bottles $3 \times$. Replicate HDPE bottles were immediately frozen and returned to the shore, where within one week they were thawed and measured by autoanalysis using the Technicon instrument. As with the freezing experiments, statistical analysis of each of the experiments on filtering samples was accomplished using Model II linear regression.

\subsection{Experiments on the long-term frozen storage of samples}

We performed experiments on $\mathrm{N}+\mathrm{N}$, SRP and SRSi to determine if the long-term frozen storage of seawater samples would affect nutrient concentrations beyond any effect of the freezing process itself. From three depths at Station ALOHA, a large number of replicate samples were collected and immediately frozen. Several were measured using the Technicon autoanalyzer after 4-day frozen storage to establish the initial nutrient concentrations and variability. At approximately monthly intervals thereafter, an individual sample from each depth was thawed and re-analyzed, until no more samples remained. Statistical analysis of the $\mathrm{N}+\mathrm{N}$, SRP and SRSi long-term frozen storage experiments was accomplished with a Student's $t$-test (Sokal and Rohlf, 1981), by comparing the set of initial replicates with the combined replicates from later time points.

\subsection{Experiments on silicate polymerization}

Additional SRSi data represent a compilation of several experiments designed to determine the extent of silicate polymerization due to freezing. The experiments compare frozen and refrigerated samples collected throughout the water column at Station ALOHA and analyzed on shore within one week of their collection. Because the "control" samples were not analyzed immediately but rather kept at $4^{\circ} \mathrm{C}$ for several days, these latter experiments do not directly assess the fresh vs. frozen question, but instead focus on the polymerization problem. The Technicon auto- analyzer was used for the SRSi determinations in these experiments, and statistical analysis was accomplished using Model II linear regression.

\section{Results}

\subsection{Experiments on the effects of freezing samples}

Model II regression analyses of the frozen onto the fresh results reveal no significant deviation from a 1:1 correspondence based on the $95 \%$ confidence intervals of slope and $y$-intercept for $[\mathrm{N}+\mathrm{N}]$ and [SRP] measured by autoanalysis (Fig. 1; Table 1). In addition, only one $\mathrm{N}+\mathrm{N}$ and one SRP sample yield concentrations after freezing that differ from the fresh values by more than twice the stated analytical precisions of the Alpkem autoanalyses. The slope for the frozen vs. fresh [SRSi] analysis does show a significant deviation from unity. Inspection of the data (Fig. 1) reveals that this result is due to slight losses in [SRSi] after freezing at the high end of the concentration range $(>120 \mu M)$. When stored for one week refrigerated, however, no difference from a $1: 1$ correspondence with the fresh [SRSi] values is noted, nor do any individual refrigerated values differ from the fresh values by more than twice the analytical precision of the measurement.

The low-level chemiluminescent $[\mathrm{N}+\mathrm{N}]$ results show a slight significant tendency toward higher values in the frozen samples than in the fresh samples (Fig. 2; Table 1), yet the greatest difference exhibited between frozen and fresh samples is only $5.6 \mathrm{n} M$. While this difference is slightly more than twice the analytical precision of the chemiluminescent technique, it is well below the precisions of autoanalytic methods. No significant deviation from a 1:1 correspondence is seen in the low-level [SRP] experiment (Fig. 2; Table 1). Some loss of precision after freezing is evident for the low-level SRP, as 4 of $16 \mathrm{SRP}$ samples exhibit concentration changes after freezing of more than twice the precision of the MAGIC analysis; however, the largest difference noted between fresh and frozen replicates in this analysis is $37 \mathrm{nM}$, less than twice the precision of either autoanalytic method. 


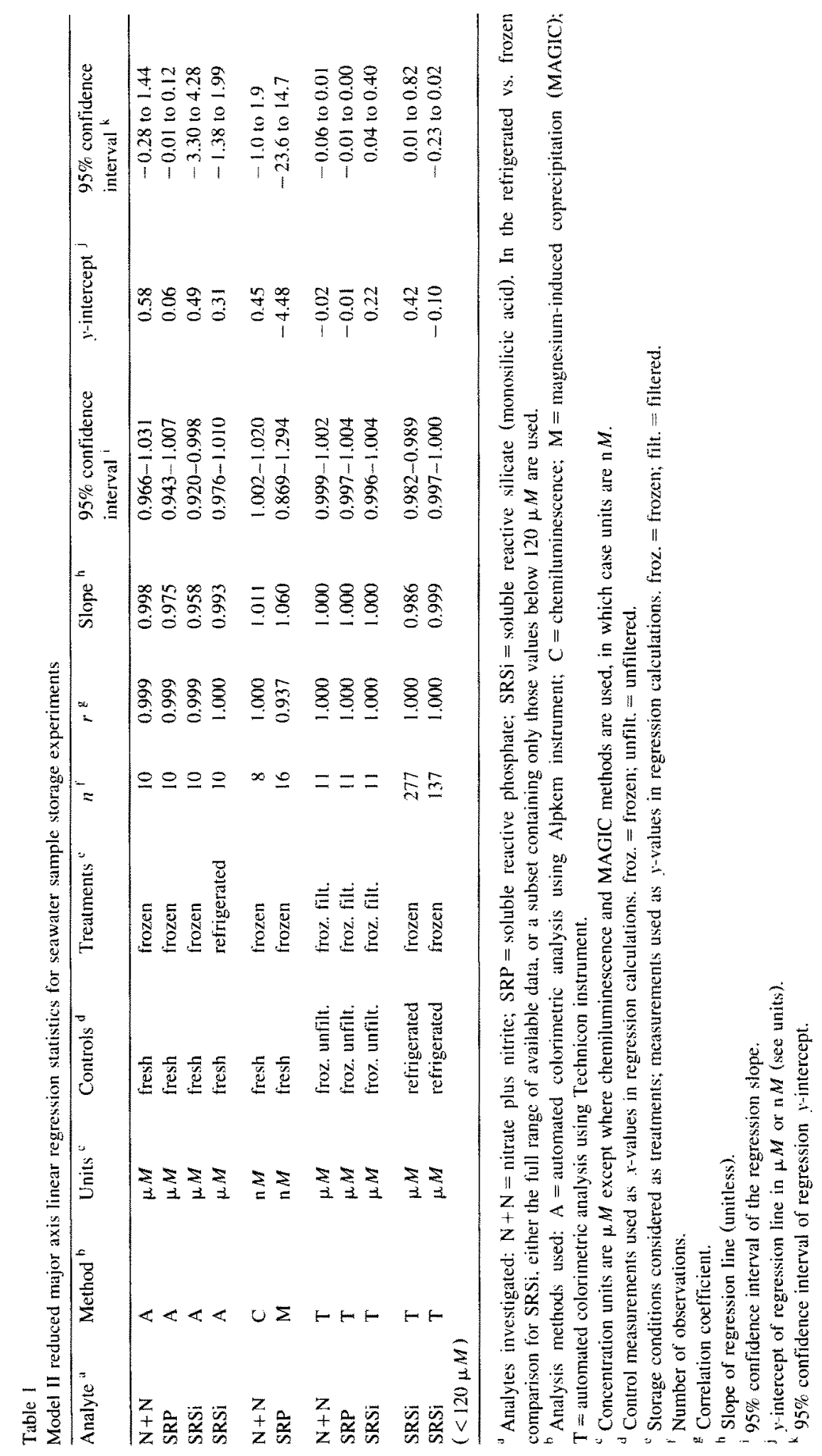




\subsection{Experiments on the filtration of samples}

Model II regression analyses of the filtered onto the unfiltered results reveal no significant deviation from a 1:1 correspondence using the $95 \%$ confidence intervals of slope and intercept for $[\mathrm{N}+\mathrm{N}]$ and [SRP] (Fig. 3; Table 1). A slight positive intercept for SRSi suggests leaching of silicate from the glass fiber filters. Because the volume filtered is constant, this amount tends to be constant, as indicated by the

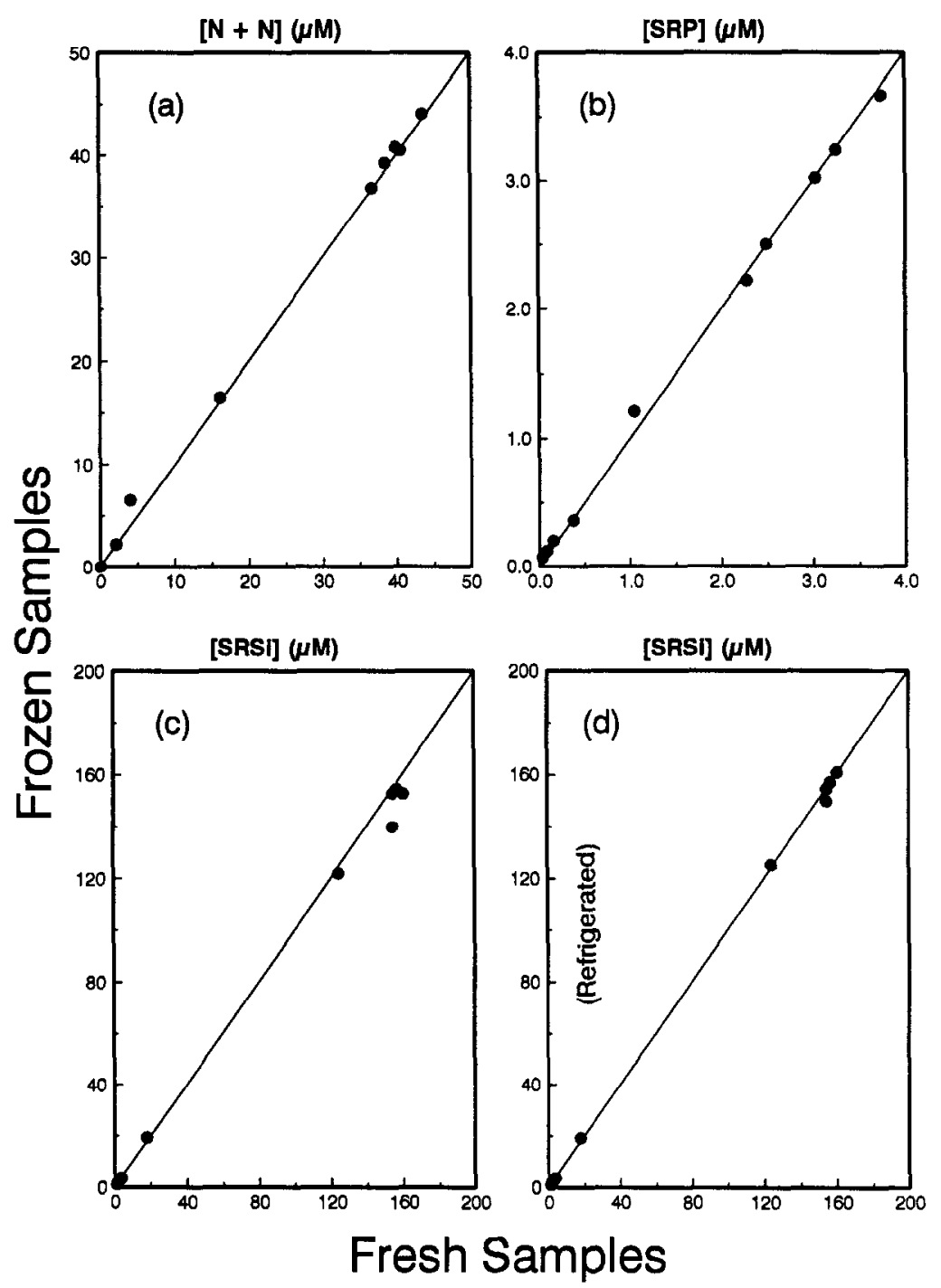

Fig. 1. a-c. Plots of frozen vs. fresh concentration determinations on unfiltered samples as determined using the Alpkem autoanalyzer: (a) nitrate I nitrite; (b) soluble reactive phosphate; and (c) soluble reactive silicatc. d. Refrigerated vs. fresh determinations of soluble reactive silicate.

The solid line in each plot represents a perfect 1:1 relationship for comparison. Linear regression statistics are listed in Table 1.

near-perfect slope of the regression line. None of the SRP samples $(n=11)$ or SRSi samples $(n=11)$, and only one of the $\mathrm{N}+\mathrm{N}$ samples $(n=11)$ deviate from the 1:1 line by more than twice the analytical precision of their respective autoanalyses.

\subsection{Experiments on the long-term frozen storage of samples}

The results of the long-term frozen storage experiments for $[\mathrm{N}+\mathrm{N}],[\mathrm{SRP}]$ and [SRSi] indicate no 


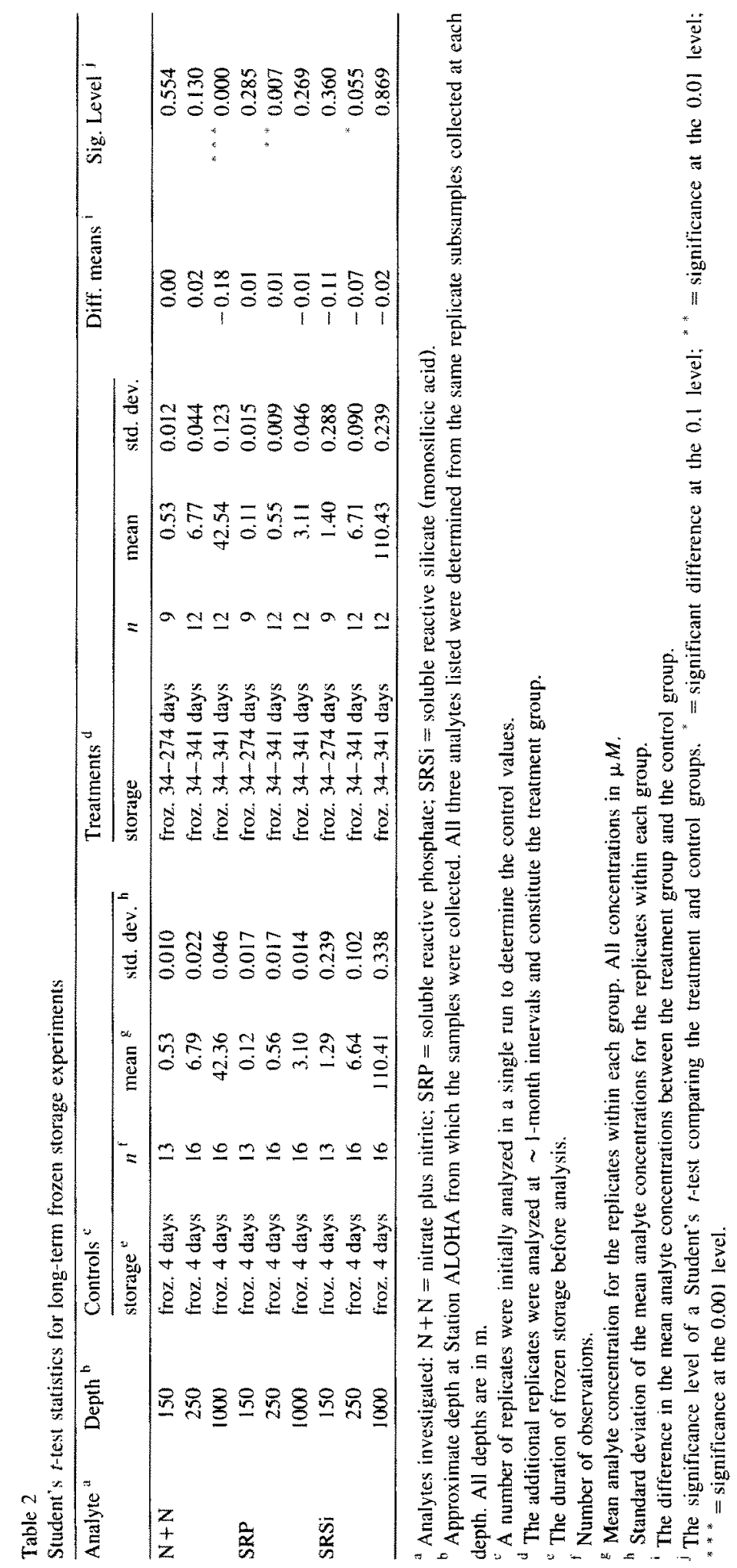




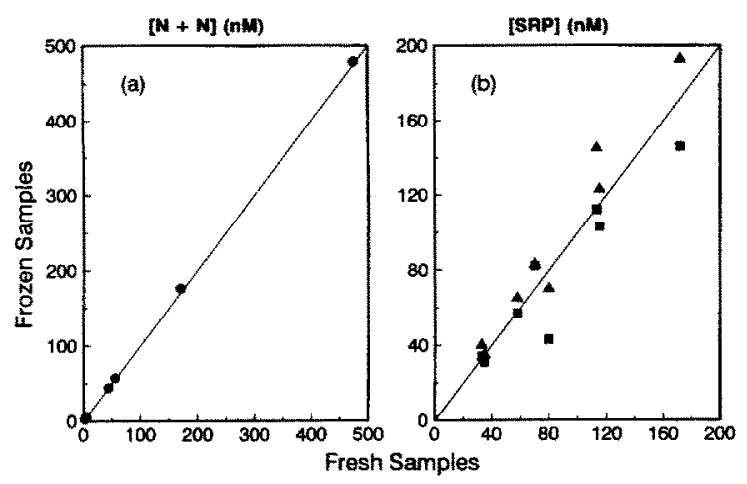

Fig. 2. Plots of frozen vs. fresh concentration determinations on unfiltered samples using low-level methods: (a) nitrate + nitrite determined by chemiluminescence; and (b) soluble reactive phosphate determined by MAGIC. Triangles in (b) indicate SRP samples frozen 1 week, squares indicate samples frozen 2 weeks. The solid line in each plot represents a perfect 1:1 relationship for comparison. Linear regression statistics are listed in Table 1. between means found significant are all less than twice the analytical precisions of the respective autoanalyses.

\subsection{Experiments on silicate polymerization}

As with the frozen vs. fresh [SRSi] comparison, the larger frozen vs. refrigerated [SRSi] results show a significant deviation from a 1:1 correspondence (Fig. 5; Table 1), produced by losses at the high end of the concentration range (Fig. 5). When only values of [SRSi] below $120 \mu M$ are considered, there is no significant difference from the 1:1 line. Furthermore, in only 1 of 137 sample pairs with [SRSi] $<120 \mu M$ was the frozen value different from the refrigerated value by more than twice the analytical precision of the Technicon autoanalyzer.

\section{Discussion}

Our results indicate that for oligotrophic oceanic waters, slow freezing of unfiltered samples in HDPE significant deviation from the initial values for periods of up to one year (Fig. 4), except for three sample sets (Table 2). These conclusions are based on a Student's $t$-test of the initial replicates with the grouped time $>0$ replicates. The three differences
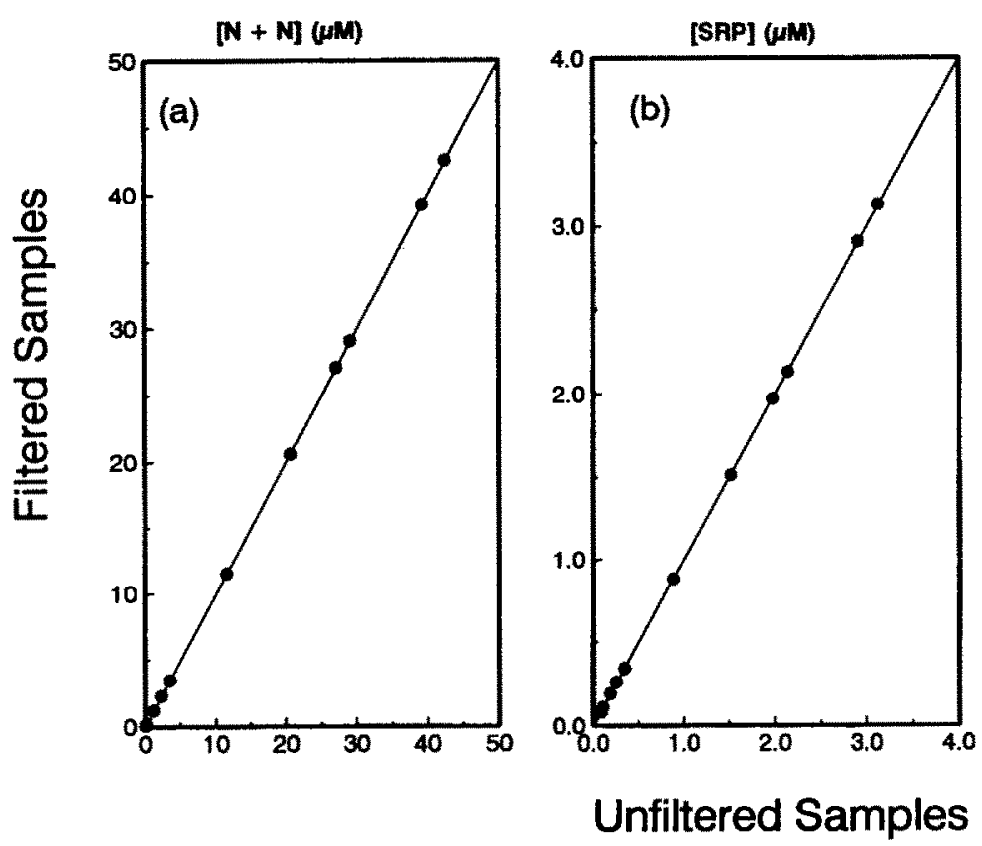

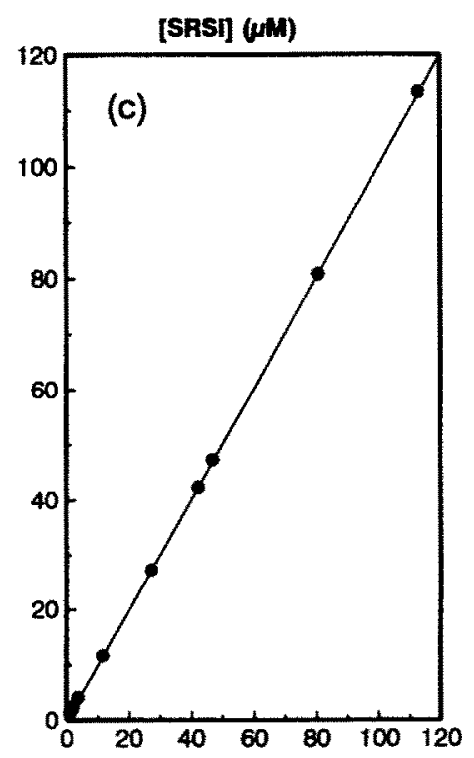

Fig. 3. Plots of filtered vs, unfiltered concentration determinations (all samples frozen) using the Technicon autoanalyzer: (a) nitrate + nitrite; (b) soluble reactive phosphate; and (c) soluble rective silicate. The solid line in each plot represents a perfect $1: 1$ relationship for comparison. Linear regression statistics are listed in Table 1. 
(a)

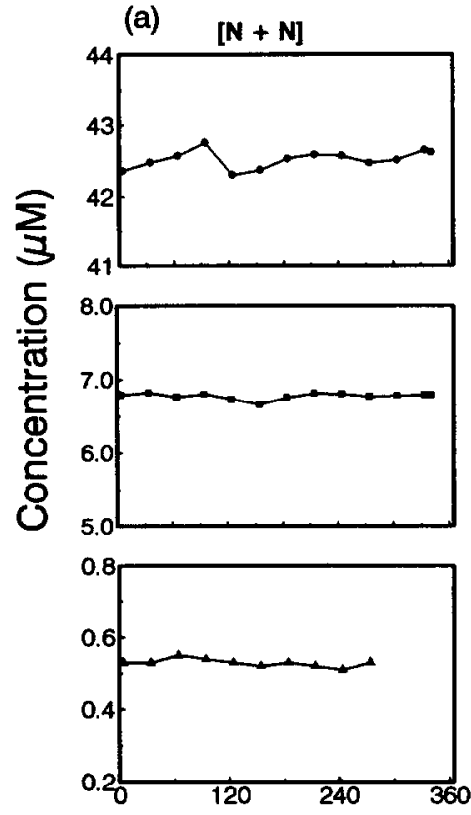

(b)
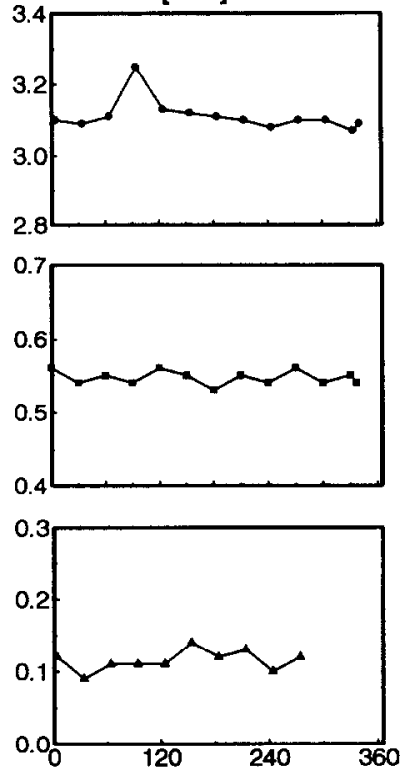

(c)

[SRS!]
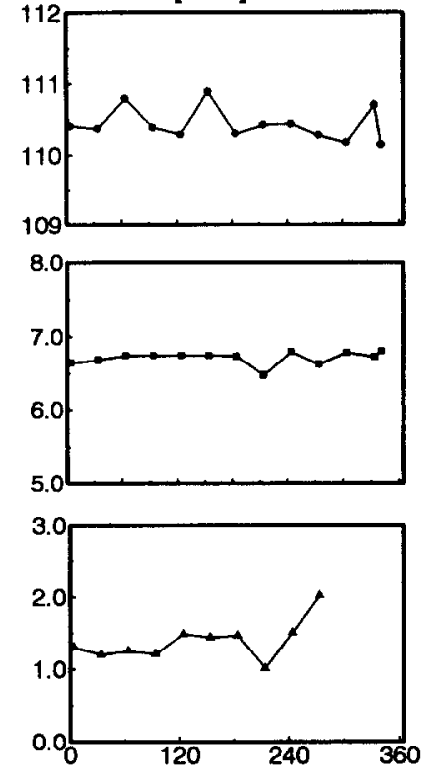

\section{Storage Time (days)}

Fig. 4. Results of long-term frozen storage experiments: time-course of analyte concentration during frozen storage as determined using the Technicon autoanalyzer: (a) nitrate + nitrite; (b) soluble reactive phosphate; and (c) soluble reactive silicate. For each constituent, results from samples collected at 1000,250 and $150 \mathrm{~m}$ are shown (top to bottom, respectively). Student's $t$-test statistics are listed in Table 2 .

bottles is an adequate preservation method for all of the analytes tested. The only experiments which reveal consistent significant deviation in concentra- tion from control values of a magnitude more than twice the estimated analytical precision are the lowlevel SRP frozen vs. fresh comparison (Fig. 2; Table

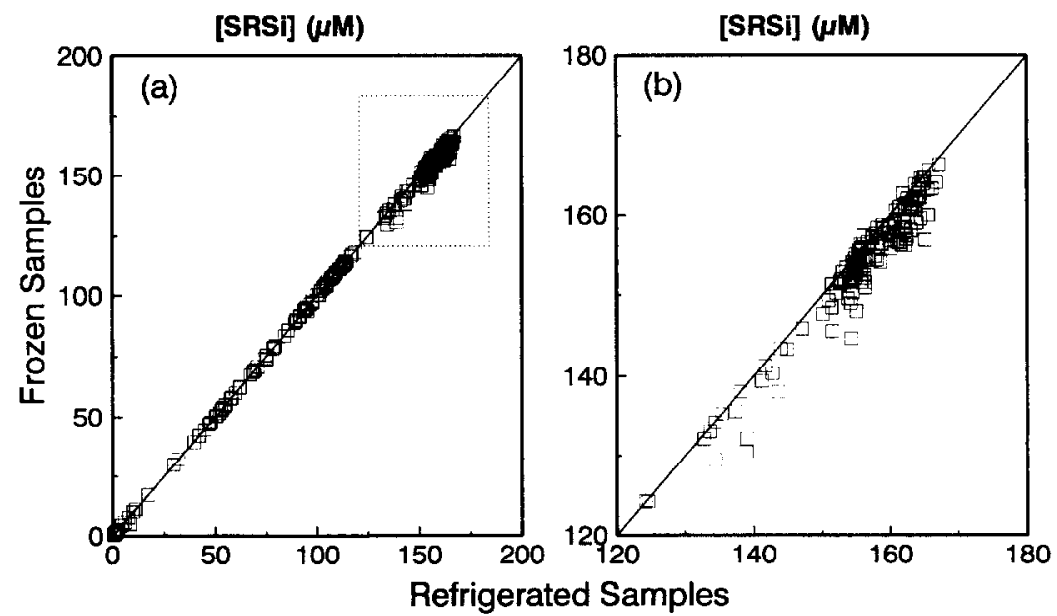

Fig. 5. Plot of frozen vs. refrigerated concentration determinations on unfiltered samples: (a) soluble reactive silicate as determined using the Technicon autoanalyzer, full range of data shown; and (b) only values $>120 \mu M$ shown to reveal polymerization losses. The solid line in each plot represents a perfect 1:1 relationship for comparison. Linear regression statistics are listed in Table 1. 
1) and the full range SRSi frozen vs. refrigerated comparison (Fig. 5; Table 1). In the case of the low-level SRP, while the results suggest a slight increase in variability caused by freezing, they also show that this effect is of a magnitude small enough to be neglected when using standard manual or automated colorimetric methods. The low-level frozen vs. fresh $\mathrm{N}+\mathrm{N}$ comparison similarly reveals that the extremely low surface concentrations in oligotrophic waters can be maintained to within a few $\mathrm{n} M$ of their original concentrations through the use of frozen storage. These results agree well with our previous results on $\mathrm{NO}_{2}^{-}$(Dore and Karl, 1996), in which the maximum concentration difference between frozen and fresh samples analyzed by chemiluminescence was $2.1 \mathrm{n} M$.

The silicate "problem" is restricted in these waters to samples with high SRSi concentrations, as evidenced by the lack of significant effect when only $<120 \mu M$ samples are tested (Fig. 5; Table 1). The polymerized silicate in the frozen high-concentration samples can be reconverted to SRSi by prolonged thawing and/or warming of the sample (Walsh, 1989; Sakamoto et al., 1990). Alternatively, if the time lag between collection and analysis is to be short, replicate sample splits can be refrigerated instead of being frozen, as evidenced by our experiments on refrigerated vs. fresh [SRSi] (Fig. 1; Table 1). As [SRSi] at Station ALOHA only exceeds 120 $\mu M$ below $\sim 1000$-m depth, no such measures need be taken for epi- and mesopelagic samples at this site; a similar conclusion can be drawn for most of the world ocean. In these cases, all three major nutrients may be adequately preserved within a single sample by freezing.

While it is of course preferable to analyze frozen samples as soon as possible upon return to the shore-based laboratory, our long-term storage results indicate that no changes greater than twice the analytical variability for the three analytes tested are evident in nutrient samples stored for nearly one year (Fig. 4; Table 2). Also, no pre-filtration of samples appears necessary in these low-particulate waters (Fig. 3; Table 1).

One reason that freezing may have been dismissed in the past by some investigators is the wide array of water types that have been used in preservation studies. Our endorsement of freezing for nutrient preservation is restricted to oligotrophic oceanic seawater, such as that found at open-ocean sites like Station ALOHA. Preservation of nutrients in freshwater, anoxic water or water with low $\mathrm{pH}$ may be hampered by precipitate formation and/or silicate polymerization, and measurements of nutrients in waters with high suspended loads may suffer from adsorption problems, turbidity interferences and rapid biological alterations. If working in these environments, investigators are advised to assess the validity of their preservation methods. Nevertheless, because oligotrophic waters are the principal component of the global ocean, our results should be applicable to a large number of current and future oceanographic investigations.

\section{Conclusions}

Inorganic nutrient concentrations in oligotrophic oceanic seawater samples are adequately maintained for several months if immediately frozen and stored at $-20^{\circ} \mathrm{C}$ in clean high-density polyethylene bottles. Of all the analytes tested, only low-level SRP samples and SRSi samples with concentrations $>120$ $\mu M$ are appreciably affected by the freezing process. The effect on [SRP] is to increase variability, but not to bias the result in one direction or the other. Furthermore, the effect on [SRP] is small enough to only be detectable using sensitive low-level techniques, and would be lost in the normal analytical variability of standard techniques. The losses of [SRSi] upon freezing samples with high concentration, while significant, are restricted to very deep waters, > 1000 III at Station ALOHA. This effect is also potentially reversible by prolonged thawing of the sample.

\section{Acknowledgements}

We thank T. Walsh for his numerous high-quality nutrient analyses, without which this work would not have been possible. We also would like to thank all of the members of the Hawaii Ocean Time-series project for their support with sampling and logistics, $\mathrm{R}$. Letelier for the use of his regression program, and the captains and crews of the research vessels Moana 
Wave and Kaimalino for their seagoing assistance. This work was supported by NSF grants OCE8717195 (R. Lukas, P.I.), OCE88-00329 (D.M.K., P.I.) and OCE93-01368 (D.M.K., P.I.), and by a graduate fellowship from the Research Corporation of the University of Hawaii awarded to J.E.D. SOEST contribution (\#4183) and U.S.-JGOFS contribution (\#229).

\section{References}

Alpkem Corporation, 1989a. Nitrate/nitrite in seawater. Alpkem Corp., Clackamas, OR, RFA method No. A303-S170-29, 6 pp.

Alpkem Corporation, 1989b. Ortho-phosphate in seawater. Alpkem Corp., Clackamas, OR, RFA method No. A303-S200-14, $11 \mathrm{pp}$.

Alpkem Corporation, 1989c. Silicate in seawater. Alpkem Corp., Clackamas, OR, RFA ${ }^{\text {s }}$ method No. A303-S220-10, 6 pp.

Ambe, M., 1978. Note of the experience in the preparation of CSK standard solutions and the ICES-SCOR intercalibration experiment, 1969-1970. Mar. Chem., 6: 171-178.

APHA (American Public Health Association), 1976. Standard Methods for the Examination of Water and Wastewater. Am. Public Health Assoc., Washington, DC, 14th ed., 1193 pp. (see especially pp. 424-425).

Armstrong, F.A., Stearns, C.R. and Strickland, J.D.H., 1967. The measurement of upwelling and subsequent biological pro cesses by means of the Technicon Autoanalyzer and associated equipment. Deep-Sea Res., 14: 381-389.

Burton, J.D., Leatherland, T.M. and Liss, P.S., 1970. The reactivity of dissolved silicon in some natural waters. Limnol. Oceanogr., 15: 473-476.

Chapman, P. and Mostert, S.A., 1990. Does freezing of nutrient samples cause analytical errors? S. Afr. J. Mar. Sci., 9: $239-247$.

Charpiot, R., 1969. Technique de conservation des échantillons d'eau de mer pour le dosage de phosphates, nitrites, nitrates, silicc ct borc. Cah. Ocćanogr., 21: 773-793.

Clementson, L.A. and Wayte, S.E., 1992. The effect of frozen storage of open-ocean seawater samples on the concentration of dissolved phosphate and nitrate. Water Res., 26: 1171-1176.

Collier, A.W. and Marvin, K.T., 1953. Stabilization of the phosphate ratio of sea water by freezing. Fish. Bull. U.S., 79: $71-76$.

Cox, R.D., 1980. Determination of nitrate and nitrite at the parts per billion level by chemiluminescence. Anal. Chem., 52: 332-335.

Dore, J.E., 1995. Microbial nitrification in the marine euphotic zonc: rates and rclationships with nitrite distributions, recycled production and nitrous oxide generation. Ph.D. Dissertation, University of Hawaii at Manoa, Honolulu, HI, $194 \mathrm{pp}$.

Dore, J.E. and Karl, D.M., 1996. Nitrite distributions and dynamics at Station AlOHA. Deep-Sea Res., II, 43: 385-482.
Garside, C., 1982. A chemiluminescent technique for the determination of nanomolar concentrations of nitrate and nitrite in seawater. Mar. Chem., 11: 159-167.

Jolicoeur, P. and Mossimann, J.E., 1968. Intervalles de confidence pour la pente de l'axe majeur d'une distribution normale bidimensionnelle. Biom. Praxim., 9: 121-140.

Karl, D.M. and Lukas, R., 1996. The Hawaii Ocean Time-series (HOT) program: Background, rationale and field implementation. Deep-Sea Res., II, 43: 129-156.

Karl, D.M. and Tien, G., 1992. MAGIC: A sensitive and precise method for measuring dissolved phosphorus in aquatic environments. Limnol. Oceanogr., 37: 105-116.

Karl, D.M., Christian, J.C., Dore, J.E., Hebel, D.V., Letelier, R.M., Tupas, L.M. and Winn, C.D., 1996. Seasonal and interannual variability in primary production and particle flux at Station ALOHA. Deep-Sea Res., II, 43: 539-568.

Kremling, K. and Wenck, A., 1986. On the storage of dissolved inorganic phosphate, nitrate and reactive silicate in Atlantic Ocean water samples. Meeresforschung, 31: 69-74.

Lewis, M.R., Harrison, W.G., Oakey, N.S., Hebert, D. and Platt, T., 1986. Vertical nitrate fluxes in the oligotrophic ocean. Science, 234: 870-873.

Macdonald, R.W., McLaughlin, F.A. and Wong, C.S., 1986. The storage of reactive silicate samples by freezing. Limnol. Oceanogr., 31: 1139-1142.

Martin, J.H., Knauer, G.A., Karl, D.M. and Broenkow, W.W., 1987. VERTEX: carbon cycling in the Northeast Pacific. Deep-Sea Res., 34: 267-285.

Morse, J.W., Hunt, M., Zullig, J., Mucci, A. and Mendez, T., 1982. A comparison of techniques for preserving dissolved nutrients in open ocean seawater samples. Occan Sci. Eng., 7: $75-106$.

Murphy, J. and Riley, J.P., 1962. A modified single solution method for the determination of phosphate in natural waters. Anal. Chim. Acta, 27: 31-36.

Ricker, W.E., 1973. Linear regression in fishery research. J. Fish. Res. Board Can.. 30: 409-434

Ricker. W.E., 1975. A note concerning Professor Jolicoeur's comments. J. Fish. Res. Board Can., 32: 1494-1498.

Ryle, V.D., Mueller, H.R. and Gentien, P., 1981. Automated analysis of nutrients in tropical sea waters. Aust. Inst. Mar. Sci. Tech. Bull., Oceanogr. Ser. No. 3, 24 pp.

Sakamoto, C.M., Friederich, G.E. and Codispoti, L.A., 1990. MBARI procedures for automated nutrient analyses using a moditied Alpkem Series 300 Rapid Flow Analyzer. Monterey Bay Aquarium Res. Inst., Tech. Rep. No. 90-2, 84 pp.

Sokal, R.R. and Rohlf, F.J., 1981. Biometry. W.H. Freeman, San Francisco, CA, 2nd ed., 859 pp.

Strickland, J.D.H. and Parsons, T.R., 1972. A Practical Handbook of Seawater Analysis. Bull. Fish. Res. Board Can., 167 (2nd edn.), $310 \mathrm{pp}$.

Technicon Industrial Systems, 1973. Orthophosphate in water and seawater. Technicon Ind. Systems, Tarrytown, NY, Autoanalyzer II ${ }^{\text {Ho }}$ Industrial Method No. 155-71W, 3 pp.

Technicon Industrial Systems, 1977. Silicates in water and seawater. Technicon Ind. Systems, Tarrytown, NY, Autoanalyzer II Industrial Method No. 186-72W, 2 pp. 
Technicon Industrial Systems, 1979. Nitrate and nitrite in water and seawater. Technicon Ind. Systems, Tarrytown, NY, Autoanalyzer $\mathrm{II}^{\text {ij }}$ Industrial Method No. 158-71W, 4 pp.

Truesdale, V.W. and Smith, C.J., 1975. The formation of molybdosilicic acids from mixed solutions of molybdate and silicate. Analyst (London), 100: 203-212.

Venrick, E.L. and Hayward, T.L., 1985. Evaluation of some lechniques for preserving nutrients in stored seawater samples. Rep. Calif. Coop. Oceanic Fish. Invest., 26: 160-168.

Walsh, T.W., 1989. Total dissolved nitrogen in seawater: a new high-temperature combustion method and a comparison with photo-oxidation. Mar. Chem., 26: 295-311.

York, D., 1966. Least-squares fitting of a straight line. Can. J. Phys., 44: 1079-1086. 